\title{
Evaluation of two Countdown Pedestrian Signal displays for pedestrian safety
}

\author{
S. A. Arhin ${ }^{1}$, E. C. Noel $^{1} \&$ M. Lakew ${ }^{2}$ \\ ${ }^{1}$ Howard University, USA \\ ${ }^{2}$ District Department of Transportation, USA
}

\begin{abstract}
The Manual on Uniform Traffic Control Devices (MUTCD) states that Countdown Pedestrian Signals (CPS) shall only be displayed during the clearance interval (FLASHING DON'T WALK - FDW) of pedestrian signals. The purpose of the CPS display is to inform pedestrians of the remaining time for crossing a road controlled by the signal, to discourage them from starting when there is insufficient time for complete crossing, and to inform those who are already on the way of the remaining time before the beginning of the DON'T WALK (DW) interval. The general literature on CPS is conclusive that CPS is better understood than the conventional pedestrian signals. In the District of Columbia the CPS display starts at the onset of the Steady WALK (SW) interval and continues through the FDW interval. It is not certain whether this CPS display has any advantage over the standard display as prescribed by the MUTCD. In this research a comparative field study of both types of countdown displays at twenty-five (25) intersections in the District of Columbia was conducted in addition to an attitudinal survey to gauge the public's preference of the type of display. The results of the evaluation showed that at the majority of the intersections studied, there were no statistically significant differences in pedestrian crossing behaviors (using 5\% significance level) due to the type of CPS display. The attitudinal survey results showed that the majority of pedestrians $(\sim 86 \%)$ and drivers $(\sim 83 \%)$ prefer CPS display which starts at the onset of the SW.
\end{abstract}

Keywords: pedestrian safety, Countdown Pedestrian Signals. 


\section{Introduction}

In recent years, there has been the accelerated use of Countdown Pedestrians Signals (CPS) at signalized intersections in the United States. A considerable number of signalized urban and sub-urban intersections in the United States are now equipped with CPS. The use of intersections by pedestrians and vehicles pose a potential conflict in the movement of the two traffic modes. Consequently, a pedestrian signal is used to allocate the right-of-way for the safe passage of pedestrians at signalized intersections.

According to the MUTCD [1], "a pedestrian signal provides a dedicated phase during which the pedestrian can enter the intersection during the steady WALK interval, and complete crossing the street during the FLASHING DON'T WALK (FDW) or STEADY DON'T WALK intervals". A CPS flashes continuously while displaying the number of seconds remaining during the pedestrian change interval, counting down to zero. The time displayed by the CPS serves as a risk mitigation mechanism used by pedestrians in resolving the crossing challenge. While the time information displayed by the signals has unanimously been accepted as a useful aid in enhancing pedestrian safety at crosswalks of signalized intersections, the type of CPS display has differed among some jurisdictions in the United States. Some jurisdictions activate their countdown display during the "STEADY WALK" (SW) interval, while others prefer to begin the countdown display during the FDW interval. The national standard on the use of CPS is provided in Section 4E-07 of the MUTCD [1]. The MUTCD prescribes that the CPS display should begin at the onset of the FDW interval. The District of Columbia CPS display studied in this research starts at the onset of the SW interval and continues through the FDW interval.

The primary purpose of CPS is to inform pedestrians of the remaining time for crossing a signalized intersection, to discourage them from starting, and to inform those already in the crossing process of the number of seconds remaining before the beginning of the DON'T WALK (DW) interval. The general literature on CPS, according to Farraher [2] and Botha et al. [3] is conclusive that CPS is well understood by pedestrians and motorists and has a significant advantage over conventional pedestrian signals. In the District of Columbia, the CPS display starts at the onset of the SW interval and continues through the FDW interval. This is contrary to the prescribed standard in the MUTCD. The District of Columbia elected to use this type of display on an experimental basis for fixed-time traffic signals prior to the 2009 MUTCD guideline. Noel and Arhin [4] conducted a study in the District of Columbia and found that the SW-FDW countdown is well-understood by pedestrians. However, it is not certain whether the SW-FWD countdown display has any advantage over the standard CPS display as prescribed by the MUTCD. This research is aimed at investigating whether the CPS display at fixed-time control intersection in the District of Columbia has any advantage over the standard display prescribed by the MUTCD. The study also includes an opinion survey of pedestrians and drivers regarding their perception of the CPS display options. 


\section{Literature review}

Pedestrian signals approved by the MUTCD [1] consist of the illuminated words WALK (or a symbol of a person) and "DON'T WALK" (DW) (or a symbolic hand). The meanings of the indications are follows:

- The Steady WALK (SW), signified by a white silhouette of a person, "means that a pedestrian facing the signal indication is permitted to start to cross the roadway in the direction of the signal indication, possibly in conflict with turning vehicles."

- The Flashing DON'T WALK (FDW), signified by a Portland orange flashing upraised hand, means that a pedestrian shall not start to cross in the direction of the indication, but a pedestrian who has already started, shall proceed out of the crosswalk.

- The Steady DON'T WALK (SDW), signified by a Portland orange steady upraised Hand, means that a pedestrian shall not enter the crosswalk in the direction of the indication.

The duration of each interval, depends on the geometric characteristics and the pedestrian and vehicular traffic at a signalized intersection. According to the 2009 edition of the MUTCD (Section 4E.07) [1], the CPS shall display the number of seconds remaining until the termination of the pedestrian change interval. The MUTCD also states that the countdown display shall neither be used during the walk interval nor during the yellow change interval of a concurrent vehicular phase. In practice, the choice of the interval to start the countdown display is largely dependent on the jurisdictional preferences.

Many evaluation studies on the effectiveness of the CPS in itself have been conducted across the United States. However, studies that focused primarily on the comparison of CPS displays are rare. Most of the studies have shown that pedestrians prefer either of the countdown displays over the conventional pedestrian signals. The findings of selected research efforts for evaluating countdown signals are discussed below.

Eccles [5] conducted a pedestrian study at locations with CPS to determine the effect of CPS at five intersections. The County applied the countdown only to the FDW interval. Comparisons were made between behavioral changes of pedestrians at the same location during daylight hours and in good weather. A total of 107 pedestrians were interviewed to determine their perception of CPS. Observations of pedestrian compliance with the signal and pedestrian-vehicle conflicts were also made. A student's t-test was used to analyze the data. At 3 of the 5 intersections evaluated, there were statistically significant decreases in the number of pedestrians remaining in the crosswalk when conflicting traffic received the green indication. The majority of the pedestrians surveyed correctly explained what the countdown signal phases meant.

The Technical Committee of the New England Section of the Institute of Transportation Engineers [6] conducted a "before" and "after" study on CPS that was installed at three intersections in Boston, Massachusetts. The countdown display was active for the entire "WALK" and FDW intervals, similar to the practice in the District of Columbia. The measures of effectiveness investigated 
were the number of pedestrians starting on WALK, the number of pedestrians starting on FDW, the number of pedestrians finishing during the DW, the number of pedestrians running or aborting, and the number of pedestrian-vehicle conflicts. The research concluded that countdown signals did not cause any significant improvement in the mentioned variables and in some instances actually degraded pedestrian safety.

In 1997, Chester and Hammond [7] conducted a study on a CPS which was installed at the intersection of Florida State Route 535 and Hotel Plaza Boulevard in Orlando, Florida. The purpose of that study was to evaluate pedestrian understanding of the CPS through field interviews. Surveys were conducted at random among local citizens and visitors. The selected crosswalk traversed eight lanes and measures about 140 feet in length. The countdown was applied to the entire WALK and FDW intervals. A total of 50 pedestrians were surveyed and the results indicated that $88 \%$ understood the functions of new countdown signals. The results of the survey of local citizens and visitors show high CPS comprehension levels of $91 \%$ and $81 \%$, respectively.

In summary, the literature suggests that the CPS provide pedestrians with additional information that help them to cross intersections more safely. The literature also suggests that pedestrians prefer CPS to conventional signals. However, none of the studies reviewed in this research compared the SW-FDW CPS display with the FDW CPS display. This study is aimed at determining whether the SW-FDW CPS has any safety advantage over the FWD CPS.

\section{Research methodology}

A "before" and "after" study was performed to compare the two types of CPS displays, as shown in Figure 1.

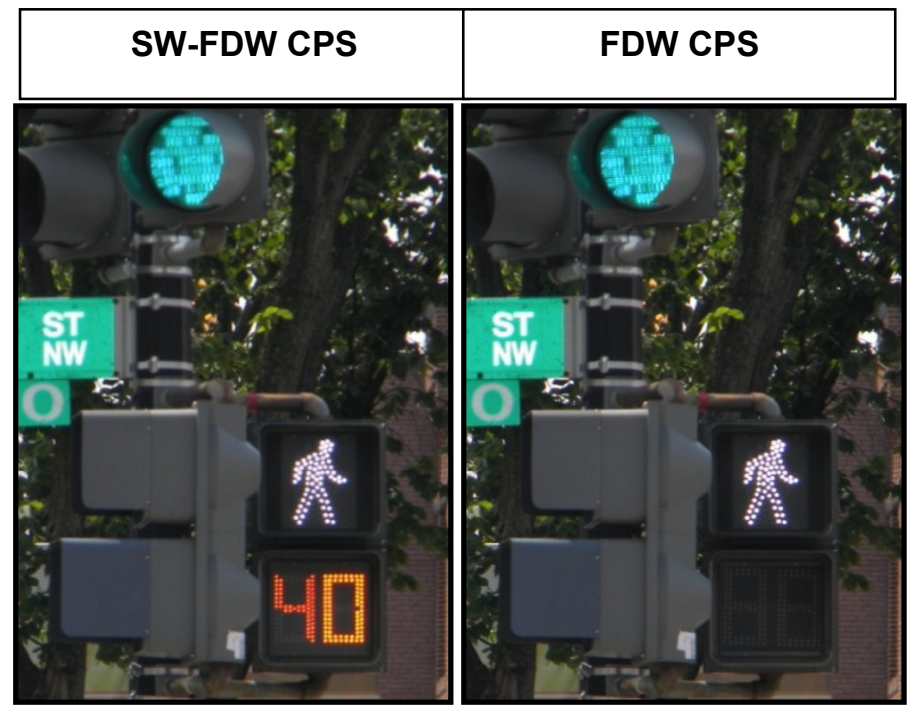

Figure 1: $\quad$ The two CPS displays. 
The "before" scenario is the SW-FDW CPS in which the countdown starts at the beginning of the SW interval and continues through the FDW interval, while in the "after" scenario the countdown coincides with the FDW interval. Twentyfive (25) intersections, which experience high pedestrian crossing activities where selected for this study. From playback of video recordings of traffic of both morning and evening peak periods, pedestrian and driver behaviors were observed at 25 crosswalks located at the 25 intersections. The same observations were made at each crosswalk during the "before" and "after" scenarios. The data collected for the two scenarios were analyzed for statistical significance using a 95\% confidence interval. In addition, a pedestrian and driver survey was conducted to evaluate their understanding and preferences for each of the two CPS displays.

The following variables were analyzed for the comparative study of SWFDW CPS and FDW CPS displays:

1. Pedestrians completing crossing during the FDW interval.

2. Pedestrians beginning to cross during FDW interval. Pedestrians are not supposed to start crossing during this interval.

3. Pedestrian-vehicle conflicts: This occurs when either a pedestrian (or group of pedestrians) take an evasive action to avoid collision with a vehicle. This study considered only conflicts that occurred between a pedestrian and a vehicle making a right-turn to/from the crosswalk.

\subsection{Attitudinal survey}

A pedestrian survey was conducted at six of the study intersections. The survey was conducted between 9:00 A.M. and 2:00 P.M. under good weather conditions. The driver survey was conducted at the offices of District Department of Motor Vehicles in the District of Columbia where drivers are easily accessible. A total of 744 pedestrians and 243 drivers were surveyed. The survey questions were posed to willing pedestrians and drivers at the respective locations. The interviewers used animated displays of the two CPS displays on laptops for illustration. Some of the survey questions that were posed presented here are as follows:

\section{For pedestrians:}

1. Which display provides you with more information?

2. Which display do you prefer for crossing signalized intersections?

\section{For drivers:}

1. Does the number in the CPS display help you make intersection driving decisions?

2. Which of the displays do you prefer?

\subsection{Statistical analysis}

The three variables defined in this study were analyzed using the test statistic of proportions. It was assumed that the samples collected at each intersection 
during the "before" and the "after" scenarios were independent and random. The sample proportions for each variable were obtained. The proportion for each variable was calculated by dividing the frequency of the outcome by the sample size. The sample size is the total number of pedestrians observed during the 2hour duration. Applying the normal approximation to the binomial to each population, the estimators of each proportion can be assumed to approximate to the normal distribution. Since the sample sizes used in this study were large $(\mathrm{N} \geq 30)$, the sampling distribution of the difference in proportions was also assumed to be normally distributed (13). A survey was conducted to obtain opinions of pedestrians and drivers in the District of Columbia regarding their preference of the CPS displays. The opinions were tallied and tabulated from which the summaries were obtained.

The null hypothesis $\left(H_{0}\right)$ and the alternative hypothesis $\left(H_{l}\right)$ were as follows:

$$
\left\{\begin{array}{l}
H_{0}: P_{b}=P_{a} \\
H_{1}: P_{b} \neq P_{a}
\end{array}\right.
$$

or

$$
\left\{\begin{array}{l}
H_{0}: P_{b}-P_{a}=0 \\
H_{1}: P_{b}-P_{a} \neq 0
\end{array}\right.
$$

where

$P_{b}=$ the proportion of the variable of interest for the SW-FDW CPS

$P_{a}=$ the proportion of the variable of interest for FDW CPS

The null hypothesis $\left(H_{o}\right)$ states that there is no difference between the observed variable for the two scenarios. Using a two-tailed test at $5 \%$ level of significance, $\left(H_{1}\right)$ would be rejected if the absolute value of $\mathrm{z}$-statistic is greater than the critical value (1.96).

\subsection{Test statistic}

The z-statistic was calculated from the following formula:

$$
z=\frac{P_{b}-P_{a}}{\sqrt{p q\left(\frac{1}{N_{b}}+\frac{1}{N_{a}}\right)}}
$$

where:

$$
p=\frac{N_{b} P_{b}+N_{a} P_{a}}{N_{b}+N_{a}} \text { and } q=1-p,
$$

$N_{b}=$ the sample size (Total number of pedestrians) for SW-FDW CPS

$N_{a}=$ the sample size (Total number of pedestrians) for FDW CPS.

$p=$ proportion of variable of interest

$q=1-p$ 


\section{Results}

\subsection{Hypothesis testing}

The analyses of the difference in proportions for the "before" and "after" scenarios were conducted for morning and evening peak periods at $5 \%$ level of significance.

\subsubsection{Pedestrians completing crossing during the FDW interval}

The proportion of pedestrians completing crossing during the FDW interval were computed. For the A.M. peak period, the proportions of pedestrians completing crossing during the FDW interval decreased at 14 intersections and increased at the remaining 11 intersections. The reductions were statistically significant at five (of the 14) intersections, and the increases were significant at four (of the 11) intersections during the A.M. peak period. In the PM peak period, 15 of the intersections experienced reductions in the proportions of pedestrians completing the crossing in the FDW interval, while 10 intersections showed an increase in proportion. The reductions in proportions were statistically significant at four (of the 15) intersections, and the increases in proportions were found to be statistically significant at five (of the 10) intersections. Overall, in the A.M. peak periods, the alternate hypothesis $H_{l}$, that there is a difference in proportions, was rejected at the nine intersections that produced statistically significant changes in proportions of pedestrians completing crossing during the FDW interval. For the P.M. peak hours, the hypothesis $H_{I}$ was rejected at nine intersections.

In summary, as shown in Table 1, the results of the hypothesis test for this variable at 5\% level of significance indicate in general that there is no discernable behavioral change in pedestrian behavior due to the type of CPS display. The majority of the intersections in both the morning and evening peak periods recorded no statistically significant difference in pedestrian behavior due to the CPS displays.

Table 1: $\quad$ Summary results for "pedestrian completing crossing during the FDW Interval".

\begin{tabular}{|c|c|c|}
\hline \multirow{2}{*}{$\begin{array}{c}\text { Peak } \\
\text { Period }\end{array}$} & \multicolumn{2}{|c|}{$\begin{array}{c}\text { Percentage of intersections showing statistically } \\
\text { significant results }\end{array}$} \\
\cline { 2 - 3 } & No difference in proportions & Difference in proportions \\
\hline Morning & $64 \%$ & $36 \%$ \\
\hline Evening & $64 \%$ & $36 \%$ \\
\hline
\end{tabular}

\subsubsection{Pedestrian starting to cross during FDW}

The same analytical process was used for this variable. The proportion of pedestrians that started crossing during the FDW was observed for the "before" and "after" scenarios. Table 2 presents the summary results of the hypothesis tests for pedestrian beginning to cross at the onset of FDW. Overall, there was no discernable behavioral change in this pedestrian behavior due to the type of CPS display, at $5 \%$ level of significance. 
Table 2: $\quad$ Summary Results for "pedestrian starting to cross during FDW".

\begin{tabular}{|c|c|c|}
\hline \multirow{2}{*}{$\begin{array}{c}\text { Peak } \\
\text { Period }\end{array}$} & \multicolumn{2}{|c|}{$\begin{array}{r}\text { Percentage of intersections showing statistically } \\
\text { significant results }\end{array}$} \\
\cline { 2 - 3 } & No difference in proportions & $\begin{array}{c}\text { Difference in } \\
\text { proportions }\end{array}$ \\
\hline Morning & $52 \%$ & $48 \%$ \\
\hline Evening & $64 \%$ & $36 \%$ \\
\hline
\end{tabular}

\subsubsection{Pedestrian-vehicle conflicts}

The results for the evaluation of pedestrian-vehicle conflicts, using the same procedure, are presented in Table 3. In summary, as shown in Table 3, the results of the hypothesis tests for this variable indicate in general that there was no discernable behavioral change in pedestrian-vehicle conflicts due to the type of CPS display.

Table 3: $\quad$ Summary results for "pedestrian-vehicle conflicts".

\begin{tabular}{|c|c|c|}
\hline \multirow{2}{*}{$\begin{array}{c}\text { Peak } \\
\text { Period }\end{array}$} & \multicolumn{2}{|c|}{$\begin{array}{c}\text { Percentage of intersections showing statistically } \\
\text { significant results }\end{array}$} \\
\cline { 2 - 3 } & No difference in percentage & $\begin{array}{c}\text { Difference in } \\
\text { percentage }\end{array}$ \\
\hline Morning & $72 \%$ & $28 \%$ \\
\hline Evening & $68 \%$ & $32 \%$ \\
\hline
\end{tabular}

\subsection{Pedestrian survey}

A survey, designed to gather information from pedestrians regarding their understanding and preference for the two types of CPS, was conducted at six intersections where SW-FDW countdown display was changed to FDW countdown display. Pedestrians were selected at random from those who were waiting at the curb to cross at the intersections. The total number of respondents was 744 .

\subsubsection{Results for question 1}

The summary of the responses to the question "As a pedestrian, which display provides you with more information?" is presented in Table 4.

The results indicate that approximately $90 \%$ of the pedestrians surveyed chose the SW-FDW countdown which corresponds to the "before" scenario. The responses suggest that pedestrians believe that displaying the time during the SW interval help them make more educated crossing decisions.

Table 4: $\quad$ Responses to question 1 of pedestrian survey.

\begin{tabular}{|l|c|}
\hline \multicolumn{1}{|c|}{ CPS Display Type } & $\begin{array}{c}\text { Percentage of } \\
\text { Response }\end{array}$ \\
\hline SW Plus FDW & $90.2 \%$ \\
\hline FDW Only & $9.8 \%$ \\
\hline
\end{tabular}




\subsubsection{Results for question 2}

Finally, pedestrian were asked to indicate which of the two types of CPS displays they would prefer for signalized intersections in the City. The summary of the responses to that question is presented in Table 5. Approximately $86 \%$ of the respondents said that they prefer the SW-FDW countdown display to be used at signalized intersection in the City.

Table 5: Responses to question 2 of pedestrian survey.

\begin{tabular}{|l|c|}
\hline \multicolumn{1}{|c|}{ CPS Display Type } & $\begin{array}{c}\text { Percentage of } \\
\text { Response }\end{array}$ \\
\hline SW Plus FDW & $85.9 \%$ \\
\hline FDW Only & $14.1 \%$ \\
\hline
\end{tabular}

Some of the pedestrians surveyed made a number of comments about the FDW countdown display. The WALK interval for FDW display is usually exhibited with a SW symbol without the countdown time. Some respondents contended that when there is no countdown time below the SW, it appears to them that the pedestrian signal is malfunctioning, until the FDW interval is displayed with the countdown seconds before proceeding to cross.

\subsection{Driver survey}

A survey was conducted to assess driver's opinions about the countdown displays. A total of 243 drivers were surveyed. The following questions were posed:

1. Does the number in the CPS display help you make intersection driving decisions?

2. Which of the displays do you prefer?

Tables 6 and 7 show the results of the survey. Nearly all drivers that admitted paying attention to the countdown also agreed that the CPS help them make driving decisions. In addition, the majority (83\%) of drivers surveyed prefer SWFDW display because it gives them more information than the FDW display.

Table 6: Responses to question 1 of driver survey.

\begin{tabular}{|l|c|}
\hline \multicolumn{1}{|c|}{ CPS Display Type } & $\begin{array}{c}\text { Percentage of } \\
\text { Response }\end{array}$ \\
\hline SW Plus FDW & $99.4 \%$ \\
\hline FDW Only & $0.6 \%$ \\
\hline
\end{tabular}

Table 7: $\quad$ Responses to question 2 of driver survey.

\begin{tabular}{|l|c|}
\hline \multicolumn{1}{|c|}{ CPS Display Type } & $\begin{array}{c}\text { Percentage of } \\
\text { Response }\end{array}$ \\
\hline SW Plus FDW & $82.6 \%$ \\
\hline FDW Only & $17.4 \%$ \\
\hline
\end{tabular}




\section{Conclusions}

From the results, $36 \%$ of the intersections showed statistically significant changes in proportions (reductions or increases) of pedestrians who completed their crossing during the FWD interval in the A.M. peak period. In the P.M. peak period, $36 \%$ of the intersections recorded statistically significant changes in proportions. Overall, there is no discernable behavioral change in this pedestrian behavior due to the type of CPS display.

The evaluation of pedestrians beginning to cross during FDW showed statistically significant difference in proportions at $48 \%$ of the intersections during the A.M. peak period. The P.M. peak period showed $36 \%$ of the intersections had a statistically significant increase or decrease in proportion. These results suggest that there is no behavioral change in this pedestrian behavior due to the type of CPS display.

The results indicate that $28 \%$ of the intersections recorded statistically significant differences in proportions of pedestrians who had conflicts with vehicles in the A.M. peak period. During the P.M. peak period, intersections that had significant changes in proportions related to this behavior represented $32 \%$. Overall, only one intersection showed a decrease in proportions for both A.M. and P.M. peak periods. The increases in proportions were significant at two of the 25 intersections. In summary, there is no clear pattern of change in pedestrian-vehicle conflicts due to the type of CPS display.

The pedestrian survey showed that the majority of respondents prefer the full countdown display (SW-FDW CPS) over the FDW CPS (as prescribed in the MUTCD). These results imply that pedestrian believe that the time displayed during the WALK interval of the SW-FDW CPS help them make better crossing decisions. The driver survey results indicate that most of the drivers paid attention to CPS displays and use the countdown to make driving decisions at intersections (99\%). The majority of the surveyed drivers indicated that they prefer SW-FDW CPS since the displayed time helps them in making driving decisions at signalized intersections.

\section{Acknowledgement}

Appreciation is extended to the District Department of Transportation for supporting this study.

\section{References}

[1] Federal Highway Administration (2003). Manual on Uniform Traffic Control Devices for Streets and Highways, 2003 Edition. Washington, DC.

[2] Farraher, B.E.B. (2000). Pedestrian Countdown Indication - Market Research and Evaluation. Minnesota Department of Transportation: http:/www.dot.state.mn.us/metro/trafficeng/signals/files/compendium.pdf 
[3] Botha, J.L., Zabyshny, A.A., Day, J.E., Northouse, R.L., Rodriguez, J.O., \& Nix, T.L. (2002). Pedestrian Countdown Signals: An Experimental Evaluation - Volume 1. Retrieved October 16, 2003 from City of San Jose, California Web site: www.ci.san-jose.ca.us/dot/forms/ report_pedcountdown.pdf

[4] E. Noel, S. Arhin. Evaluation of Countdown Pedestrian Signals in the District of Columbia. Washington DC, 2006.

[5] Eccles, K.A. (2003). Evaluation of Pedestrian Countdown Signals. Silver Spring, MD: Prepared by BMI-SG for Montgomery County Maryland and Maryland State Highway Administration.

[6] Ken Petraglia. An Evaluation of Countdown Pedestrian Signals. New England Chronicle. May 2004.

[7] Chester, D.C., Hammond, M. Evaluation of Pedestrian Understanding of Pedestrian Countdown Signals. 68th Annual Meeting of Institute of Transportation Engineers, 1998. 